\title{
Non Invasive Mechanical Ventilation in Acute Respiratory Failure.
}

\author{
Asmaa Ramadan khalaf ${ }^{1}$; Azza Mahmoud Ahmad ${ }^{1}$;Hamdy Ali Mohammadien ${ }^{1}$ and Gamal \\ Mohammed Rabie ${ }^{2}$ \\ Departments of Chest Diseases and Tuberculosis, Sohag ${ }^{1}$ and Assiut ${ }^{2}$ Universities
}

\begin{abstract}
Objectives : Acute respiratory failure is a common clinical condition encountered in emergency department and intensive care units (ICU). The use of NIV during acute respiratory failure (ARF) has increased since the late 1990s for all diagnoses, including patients with and without chronic obstructive pulmonary disease (COPD). The use of NIV is associated with reduced tracheal intubation, duration of hospitalization, and mortality.
\end{abstract}

Aim of the work: To evaluates the effectiveness of Non invasive ventilation in the management of acute hypercapnic respiratory failure of different aetiologies as acute exacerbation of COPD, and acute hypoxemic respiratory failure.

Design of study: Prospective, Observational, Single-center study.

Patients and Methods: 103 patients with acute respiratory failure (ARF) were admitted to Respiratory Intensive Care Unit (RICU) of Chest Department at Assuit University Hospital and received non invasive ventilation, in all patients demographic , clinical and functional parameters were recorded including the cause of acute respiratory failure. NIV success was defined as clinical and gasometric improvement and discharge to regular ward, while need of endotrachial intubation was considered NIV failure.

Results: one hundred and three patients with mean age $\mathbf{5 9 . 1}$ years were included in the study, $62(60 \%)$ were males and $\mathbf{4 1}(\mathbf{4 0 \%})$ were females, Baseline $\mathrm{pH}, \mathrm{PaCO2}$ and $\mathrm{PaO2}$ were $\mathbf{7 . 5 1} \pm \mathbf{0 . 0 9}, \mathbf{7 2} \pm \mathbf{2 1 . 6 3}$ and $\mathbf{5 9 . 4 1} \pm \mathbf{2 0 . 3 4} \mathrm{mmHg}$ respectively.

The success rate with NIPPV was $69 \%$, with 71 of 103 patients weaned successfully. Significant improvements were observed at 2 hour,24-48hrs following institution of NIPPV in $\mathrm{pH}(\mathbf{7 . 3 4} \pm \mathbf{0 . 0 8}, \mathrm{P}<\mathbf{0 . 0 2}), \mathrm{PaCO} 2(\mathbf{6 2 . 8 7} \pm \mathbf{1 7 . 9 4}, \mathrm{P}<\mathbf{0 . 0 0 2})$ and $\mathrm{PaO}$ 2(74.30 \pm 14.45 $\mathrm{P}<\mathbf{0 . 0 0 1}$ ). These improvements maintained (within $24 \mathrm{hrs}$ ) postweaning from the ventilator, $\mathrm{pH}$ 7.39 \pm 0.04, $\mathrm{PaCO} 2$ 56.76 \pm 10.18, $\mathrm{PaO} 2$ 73.28 \pm 10.04 ( $P<0.001)$.

Duration of mechanical ventilation, length of ICU stay were significantly longer in NIV failure group $(\mathbf{p}<\mathbf{0 . 0 0 0 1})$, the complications and death were significantly higher in NIV failure group $(\mathrm{p}<\mathbf{0 . 0 0 0 0 1}),(\mathrm{p}<\mathbf{0 . 0 0 0 1})$.

Serum albumin level was significantly lower in the NIV failure group $(\mathrm{p}<\mathbf{0 . 0 1})$.

Conclusion: The use of NIV in patients presenting with ARF of diverse etiology

has shown to be effective in the improvement of clinical and gasometric parameters, in preventing endotracheal intubation, and improving overall survival .

Keywords: Acute respiratory failure, noninvasive ventilation, COPD.

\section{Introduction}

Respiratory failure (RF) is a failure respiratory system in one or both of its gas exchange functions: oxygenation and/or elimination of carbon dioxide. It's defined by $\mathbf{P a O 2}<\mathbf{6 0} \mathrm{mmHg}$ (1). Acute respiratory failure is a common clinical condition encountered in emergency department and intensive care units (ICU) (2). The utilization of noninvasive mechanical ventilation (NIV) has become one of the most important developments in the field of 
mechanical ventilation over the past two decades. The use of NIV during acute respiratory failure (ARF) has increased since the late 1990s for all diagnoses, including patients with and without chronic obstructive pulmonary disease(COPD)(3). Non-invasive positive pressure ventilation (NIPPV) works by providing pressure support that gives ventilatory assistance during inspiration, allows respiratory muscles to work less, increases the volume inspired per minute and improves arterial blood gas (ABG) levels (4). The use of NIV is associated with reduced tracheal intubation, duration of hospitalization, and mortality.(5) Most experience with noninvasive ventilation has accrued with either bilevel positive airway pressure(BiPAP) or pressure support ventilation, less so with volume ventilation and continuous positive airway pressure (CPAP), which is infrequently used as a mode of ventilatory support(6).

\section{Aim of the work:}

The study evaluates

- Task of Non invasive ventilation in management of acute hypercapnic respiratory failure of different aetiologies as acute exacerbation of COPD.

- The effectiveness of NIV in acute hypoxemic respiratory failure.

\section{Patients and Methods:}

The present prospective, observational, single-center study was conducted on 103 patients with acute respiratory failure (ARF) 62 males $(60 \%)$ and 41 females $\mathbf{( 4 0 \% )}$ ) their age ranged from 20 to 88 years with mean age $\mathbf{5 9 . 1}$ years.All patients were admitted to Respiratory Intensive Care Unit (RICU) of Chest Department at Assuit University Hospital during the period from January 2016 to December 2016 and were evaluated for the use of Non Invasive Ventilation (NIV) in acute Respiratory failure

\section{Inclusion criteria:}

Patient with acute respiratory failure with either:

-Acute Hypoxemic respiratory failure (Type 1) defined by :

a $\mathrm{PaO2}$ of $<\mathbf{6 0} \mathrm{mmHg}$ or $\mathrm{PaO2}$ to $\mathrm{FiO2}$ ratio $<200$ with a normal or low PaCO2.

-Acute on top of chronic Hypercapnic respiratory failure(Type 2 ) defined by:

a $\mathrm{PH}<\mathbf{7 . 3 5}, \mathrm{PaCO} 2$ of $>\mathbf{5 0} \mathbf{m m H g}$ and

a $\mathrm{PaO2}$ of $<\mathbf{6 0} \mathrm{mmHg}, \mathrm{HCO} 3>\mathbf{2 6}$ $\mathrm{mmol} / \mathrm{L}$

Exclusion criteria:The absolute contraindications for NIV

1. Respiratory or cardiac arrest.

2. Haemodynamic instability (Systolic B.P $<90 \mathrm{mmHg}$ and/or $\mathrm{HR}<\mathbf{5 0}$ beat/min) despite fluid challenge or use of vasopressors agents, unstable angina, acute MI, serious arrythmia.

3. Coma or seizure disorders.

4.Agitation need sedation.

5. copcious secretions that unable to be spontaneously cleared from

the airway with high risk of aspiration.

6. facial trauma, burns, or facial surgery. All admitted patients were subjected to:

1-Thorough History taking: from patients or their relatives.

Including: age, sex, occupation ,smoking status, underlying comorbidities, history of previous ICU admission.

2-Thorough Clinical Examination including:

-General examination :

Heart rate, respiratory rate ,Blood pressure(systolic and diastolic blood pressure) and temperature.

-chest examination , abdominal and cardiac examination

3-Investigations:

1. Chest radiograph: a- $\mathrm{x}$-ray posteroanterior view , b-Computed tomography when needed. 
2. chest ultrasonography (US) when needed

3. Arterial Blood Gases analysis: including ( $\mathrm{PH}, \mathrm{paO2}$,paCO2 ,SaO2)

4.other investigations: complete blood count (CBC), Blood chemistry including liver function tests, renal function tests, serum electrolytes e.g. $\mathrm{Na}+, \mathrm{K}+, \mathrm{Cl}, \mathrm{C}$ a++.

All patients were mechanically ventilated through an ICU ventilator using a dedicated NIV mode (Bennet 840 Ventilator system, Hamilton-GS, Puritan, Engström Carestation).The ventilator was connected with conventional ventilator tubing to a clear, oronasal face mask (PerformaTrak, Philips Respironics) the masks were used in all patients with suitable adult sizes. The head of the bed was raised to an angle of $45^{\circ}$ and was kept elevated during NIV to reduce the risk of aspiration. Pressure Support Ventilation (PSV) associated with Positive end-expiratory pressure(PEEP) (CPAP and PS ) is the used mode of support during NIV.

NIV setting: After the mask was attached to the patient, pressure support was adjusted and increased from 5 up to $20 \mathrm{~cm} \mathrm{H} 20$ to obtain an tidal volume of 5-7 $\mathrm{mL} / \mathrm{kg}$ of predicted body weight, a respiratory rate lower than 25 breaths per minute, attenuation of respiratory accessory muscle activity and achievement of patient's comfort. Positive endexpiratory pressure (PEEP) was initiated at $5 \mathrm{~cm}$ $\mathrm{H} 2 \mathrm{O}$ and could be increased in steps of 2 to $3 \mathrm{~cm} \mathrm{H2O}$ up to $15 \mathrm{~cm} \mathrm{H2O.}$ The fraction of inspired oxygen $\mathrm{FiO2}$ was titrated to maintain the arterial oxygen saturation $\mathrm{SpO2} \mathrm{88-92 \%}$ in type II respiratory failure and $\mathbf{9 2 - 9 5 \%}$ in type I respiratory failure. NIV was continuously applied, with every 4-6 h of continuous NIV, patients had periods of "rest", (20-30 $\mathbf{~ m i n})$ off the mask while receiving supplemental oxygen ,nebulizer, or dietary supplements with a minimal duration of $16 \mathrm{~h}$ per day until a significant clinical improvement of the patient occurred. If effective, gradual reduction of the duration of NIV could be then considered.

\section{Monitoring}

The following data were recorded for all patients:

-Clinical parameters (respiratory rate, heart rate, blood pressure,)

-Gasometric parameters

(PH,PCO2,PO2,SaO2\%,HCO3 )

Before NIV initiation. ,Two hours (2hrs) and then 24- 48hours after initiation of NIV. And $24 \mathrm{hrs}$ after weaning.

\section{Weaning from NIV}

patients were weaned from NIV when the following criteria fulfilled including

$\bullet \mathrm{RR}<24 / \mathrm{min}$

$\cdot \mathrm{HR}<110 / \mathrm{min}$

- $\mathrm{PH}>7.35$

\section{-O2sat. $\geq 90 \%$ on Fio2 $\leq 40 \%$}

The study primary outcome include either success of treatment with NIV or failure of treatment with NIV .

Successful treatment with NIV was defined according to objective and subjective criteria that reflect the patient's improvement following NIV initiation thereby avoiding intubation in these patients.

The objective criteria included

-A decrease of $\mathbf{2 0 \%}$ in respiratory rate compared with spontaneous breathing,

-An improvement in arterial blood gases with $\mathrm{PH}>\mathbf{7 . 3 5}$

-A decrease in $\mathrm{PaCO} 2$ of $\geq \mathbf{1 5 \%}$ compared with spontaneous breathing -Maintaining a $\mathrm{SaO2} \geq \mathbf{9 0 \%}$.

The subjective criteria included improvement of the patient regarding both dyspnea and comfort.

Failure is defined as failure to achieve the previous criteria of successful treatment at any point of the study or the need for intubation. 
Failure was classified into:

a) Immediate failure (within1 hour)

b) Early (1-48 hours)

c) Late failure (after $\mathbf{4 8}$ hours)

We also recorded Duration of mechanichal ventilation, ICU and hospital lengths of stay, the complications associated with noninvasive ventilation, ICU and hospital mortality .

Statistical analysis :

Statistical analysis was performed using Statistical Package for the Social Sciences (SPSS-version 20). Results were presented as a mean \pm SD for quantitative data and as frequency and percentage for qualitative data. For

\section{Results}

comparison between two groups of quantitative data, independent sample T-test was used.For comparison between more than two groups of quantitative data, ANOVA test was used (as for comparison between clinical and gasometric parameters over the $\mathbf{4 8}$ hours of the duration of the study follow up). Chi-suqare test was applied for for qualitative data to compare between groups.Differences were considered significant when $\mathrm{P}$ value <0.05. To determine the optimum cut level that best predict outcome, the data were analyzed using the receiving operating characteristic curve (ROC) curve.

Table (1): Demographic characteristics of studied population:

\begin{tabular}{|c|c|c|}
\hline Variables & NO & $(\%)$ \\
\hline $\begin{array}{l}\text { Age } \\
\text { Mean } \pm \text { SD) } \\
\text { Median (range) }\end{array}$ & \multicolumn{2}{|c|}{$\begin{array}{l}59.1 \pm 13.5 \\
60(20-88)\end{array}$} \\
\hline $\begin{array}{l}\text { Gender } \\
\text { Females } \\
\text { Males } \\
\end{array}$ & $\begin{array}{l}41 \\
62 \\
\end{array}$ & $\begin{array}{l}(39.81 \%) \\
(60.19 \%) \\
\end{array}$ \\
\hline $\begin{array}{l}\text { Smoking status } \\
\text { Current } \\
\text { Ex-smoker } \\
\text { Non smoker }\end{array}$ & $\begin{array}{l}25 \\
31 \\
47\end{array}$ & $\begin{array}{l}(24.27 \%) \\
(30.10 \%) \\
(45.63 \%)\end{array}$ \\
\hline $\begin{array}{l}\text { Smoking index } \\
\text { Mild } \\
\text { Moderate } \\
\text { Heavy }\end{array}$ & $\begin{array}{l}7 \\
5 \\
44 \\
\end{array}$ & $\begin{array}{l}(6.80 \%) \\
(4.85 \%) \\
(42.72 \%)\end{array}$ \\
\hline
\end{tabular}

one hundered and three (103) patients with acute respiratory failure (ARF) admitted to Respiratory Intensive Care Unit of Assiut university hospital with a mean age of $\mathbf{5 9 . 1} \pm \mathbf{1 3 . 5}$ years, ranges from $\mathbf{2 0}$ to $\mathbf{8 8}$ years old were enrolled in the study and the majority were males ( $60.19 \%)$. 
Table(2): Types of acute respiratory failure and Diagnosis of studied population

\begin{tabular}{|c|c|c|}
\hline Variables & No & $(\%)$ \\
\hline $\begin{array}{l}\text { Type of acute respiratory failure } \\
\text { Acute Hypoxemic } \\
\text { Acute on top of chronic Hypercapnic }\end{array}$ & $\begin{array}{l}10 \\
93\end{array}$ & $\begin{array}{l}(9.71 \%) \\
(90.29 \%)\end{array}$ \\
\hline \multicolumn{3}{|l|}{ Diagnosis and causes of ARF } \\
\hline ARDS & 1 & $(0.97 \%)$ \\
\hline Bronchial asthma & 5 & $(4.85 \%)$ \\
\hline Bronchiectasis & 2 & $(1.94 \%)$ \\
\hline COPD & 48 & $(46.60 \%)$ \\
\hline COPD with CAP & 9 & $(8.74 \%)$ \\
\hline IPF & 5 & $(4.85 \%)$ \\
\hline IPF with CAP & $\mathbf{1}$ & $(0.97 \%)$ \\
\hline IPF with PE & 1 & $(0.97 \%)$ \\
\hline Kyphoscoliosis & 2 & $(1.94 \%)$ \\
\hline OHS & 7 & $(6.80 \%)$ \\
\hline OHS with CAP & 4 & $(3.88 \%)$ \\
\hline Overlap syndrome & 13 & $(12.62 \%)$ \\
\hline Overlap syndrome with CAP & 2 & $(1.94 \%)$ \\
\hline Pneumonia & 3 & $(2.91 \%)$ \\
\hline
\end{tabular}

ARF: acute respiratory failure ,ARDS: acute respiratory distress syndrome, COPD:chronic obstructive pulmonary disease, CAP:community acquired pneumonia, IPF:idiopathic pulmonary fibrosis, PE:pulmonary embolism, OHS: obesity hypoventilation syndrome, Overlap syndrome: chronic obstructive pulmonary disease\& obstructive sleep apnea

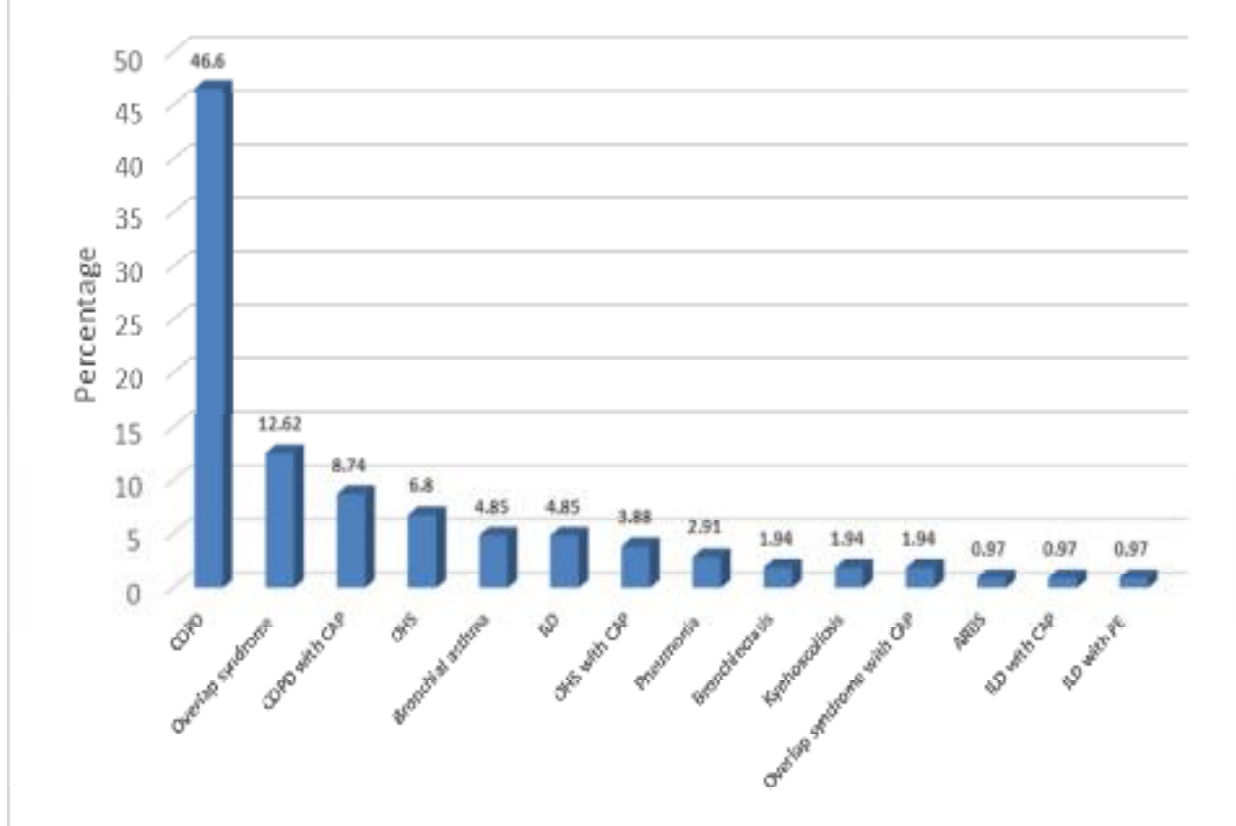

Fig.(1) : Diagnosis of studied population

As regard the type of acute respiratory failure (ARF) (93) out of (103) patients were presented with acute on top of chronic hypercapnic RF which represent the higher percentage (90.29\%) and (10) patients were presented with acute hypoxemic $\mathbf{R F}$ and as regard diagnosis of the underlying causes of acute respiratory failure the acute 
SOHAG MEDICAL JOURNAL

Vol. 21 No.1 Jan 2017
Non Invasive Mechanical Ventilation in Acute Respiratory Asmaa Ramadan khalaf

exacerbation of COPD represent the higher percentage (46.60\%) as illusterated in

Table (2), Fig (1) .

Table(3): clinical parameters of studied population before and after NIV:

\begin{tabular}{|c|c|c|c|c|c|c|}
\hline clinical parameters & Before NIV & After 2 hours & After 24-48 hours & P1 & $\mathbf{P 2}$ & P3 \\
\hline $\begin{array}{l}\text { Respiratory rate } \\
\text { Mean }\end{array}$ & $30.86 \pm 7.59$ & $24.69 \pm 5.47$ & $23.16 \pm 4.92$ & $*<0.0001$ & $*<0.0001$ & 0.23 \\
\hline $\begin{array}{l}\text { Heart rate } \\
\text { Mean }\end{array}$ & $107.12 \pm 17.38$ & $99.99 \pm 14.79$ & $99.24 \pm 14.41$ & $* 0.003$ & $* 0.001$ & 1.00 \\
\hline $\begin{array}{l}\text { Systolic blood pressu } \\
\text { Mean }\end{array}$ & $122.91 \pm 17.29$ & $120.10 \pm 17.79$ & $115.34 \pm 11.41$ & 0.61 & $* 0.002$ & 0.10 \\
\hline $\begin{array}{ll}\text { Diastolic } & \text { blo } \\
\text { pressure } & \\
\text { Mean } & \end{array}$ & $76.31 \pm 10.19$ & $74.98 \pm 10.42$ & $73.08 \pm 7.94$ & 0.25 & 0.05 & 1.00 \\
\hline $\begin{array}{l}\text { Temperature } \\
\text { Mean }\end{array}$ & $37.40 \pm 0.47$ & $37.32 \pm 0.24$ & $\mathbf{3 7 . 3 1} \pm \mathbf{0 . 3 6}$ & 0.36 & 0.20 & 1.00 \\
\hline
\end{tabular}

- $\quad$ 1 compared before NIV \& after $2 \mathrm{hrs}$

- 22 compared before NIV \& after 24-48hrs

- 33 compared After 2 hours \& after 24-48h

In Table (3) the clinical parameters of studied patients as respiratory rate ,heart rate, systolic \& diastolic blood pressures and temperature were illustrated: before NIV, 2hrs , 24-48 hrs after NIV, the results show a significant reduction of RR,HR,SBP over time after use of NIV . 
SOHAG MEDICAL JOURNAL

Vol. 21 No.1 Jan 2017
Non Invasive Mechanical Ventilation in Acute Respiratory Asmaa Ramadan khalaf

Table (4): Gasometric parameters of studied population before and after NIV use:

\begin{tabular}{|c|c|c|c|c|c|c|c|c|c|c|}
\hline $\begin{array}{l}\text { Gasometr } \\
\text { ic } \\
\text { paramete } \\
\text { rs }\end{array}$ & Before NIV & $\begin{array}{l}\text { After } 2 \\
\text { hours }\end{array}$ & $\begin{array}{l}\text { After 24- } \\
48 \text { hours }\end{array}$ & $\begin{array}{c}\text { Post } \\
\text { weaning }\end{array}$ & P1 & P2 & P3 & P4 & P5 & P6 \\
\hline $\begin{array}{l}\text { PH } \\
\text { Mean } \\
\text { Median }\end{array}$ & $\begin{array}{c}7.51 \pm 0.09 \\
7.3(7.12- \\
7.53)\end{array}$ & $\begin{array}{c}7.34 \pm 0.08 \\
7.34 \\
(7.17- \\
7.57)\end{array}$ & \begin{tabular}{|c}
$7.39 \pm 0.09$ \\
7.38 \\
$(7.17-$ \\
$7.83)$ \\
\end{tabular} & $\begin{array}{c}7.39 \pm 0.04 \\
7.38(7.34- \\
7.51)\end{array}$ & $* 0.02$ & $*<0.0001$ & $*<0.0001$ & $* 0.001$ & $* 0.001$ & 1.00 \\
\hline $\begin{array}{l}\text { PCO2(m } \\
\text { mHg) } \\
\text { Mean } \\
\text { Median }\end{array}$ & $\begin{array}{c}72 \pm 21.63 \\
72(25-113)\end{array}$ & $\begin{array}{c}67.36 \pm 18 . \\
07 \\
68(27- \\
115)\end{array}$ & $\begin{array}{c}62.87 \pm 17 \\
94 \\
66(22- \\
115)\end{array}$ & $\begin{array}{c}56.76 \pm 10.18 \\
59(32-79)\end{array}$ & 0.39 & $* 0.002$ & $*<0.0001$ & 0.45 & $* 0.001$ & 0.17 \\
\hline $\begin{array}{l}\text { PO2(mm } \\
\text { Hg) } \\
\text { Mean } \\
\text { Median }\end{array}$ & $\begin{array}{l}59.41 \pm 20.34 \\
57(21-142)\end{array}$ & $\begin{array}{c}74.30 \pm 14 \\
45 \\
73(46- \\
120)\end{array}$ & \begin{tabular}{|c}
$73.69 \pm 14$. \\
31 \\
$71(37-$ \\
$168)$
\end{tabular} & $\begin{array}{c}73.28 \pm 10.04 \\
70(54-95)\end{array}$ & $\begin{array}{c}*<0.0 \\
001\end{array}$ & $*<0.0001$ & $*<0.0001$ & 1.00 & 1.00 & 1.00 \\
\hline $\begin{array}{l}\mathrm{SaO2}(\%) \\
\text { Mean } \\
\text { Median }\end{array}$ & $\begin{array}{l}82.35 \pm 1.38 \\
86(40-99)\end{array}$ & $\begin{array}{c}92.30 \pm 8.9 \\
8 \\
93(85-99)\end{array}$ & $\begin{array}{c}92.85 \pm 6.6 \\
7 \\
94(56- \\
100)\end{array}$ & $\begin{array}{c}93.17 \pm 2.21 \\
93(87-97)\end{array}$ & 1.00 & 1.00 & $*<0.0001$ & 1.00 & $*<0.0001$ & $\begin{array}{c}*<0.00 \\
01\end{array}$ \\
\hline $\begin{array}{l}\text { HCO3(m } \\
\text { Eq/L) } \\
\text { Mean } \\
\text { Median }\end{array}$ & $\begin{array}{l}35.32 \pm 8.22 \\
36(18-60)\end{array}$ & $\begin{array}{c}36.03 \pm 8.3 \\
9 \\
36(17-57)\end{array}$ & $\begin{array}{c}35.93 \pm 8.1 \\
0 \\
37(18-53)\end{array}$ & $\begin{array}{c}35.38 \pm 9.34 \\
36(20-93)\end{array}$ & 1.00 & 1.00 & 1.00 & 1.00 & 1.00 & 1.00 \\
\hline
\end{tabular}

- p1 compared before NIV \& after $2 \mathrm{hrs}$,

- 22 compared before NIV \& after 24-48hrs

- p3 compared before NIV \& post weaning

- $\quad$ p4 compared after $2 \mathrm{hrs} \&$ after $24-48 \mathrm{hrs}$

- $\quad$ 55 compared after 2 hrs \& post weaning

- p6 compared after $24-48 \mathrm{hr} \&$ post weaning

Table (4), demonstrate the gasometric parameters(PH,PaCO2,PaO2,SaO2,HCO3) of the study patients and show a highly significant improvement 2 hrs \& 24-48hrs after NIV use \& postweaning from NIV. 
SOHAG MEDICAL JOURNAL

Vol. 21 No.1 Jan 2017
Non Invasive Mechanical Ventilation in Acute Respiratory Asmaa Ramadan khalaf

Table (5):Outcome of studied populations as regard the success and failure of NIV.

\begin{tabular}{|l||l||l||}
\hline OUTCOME & NO. & $\%$ \\
\hline \hline SUCCESS & 71 & $68.93 \%$ \\
\hline \hline FAILURE & 32 & $31.07 \%$ \\
\hline \hline Immediate Failure & 4 & $3.88 \%$ \\
\hline \hline Early Failure & 10 & $9.71 \%$ \\
\hline \hline Late Failure & 18 & $17.48 \%$ \\
\hline
\end{tabular}

Table 5 show the outcome of the studied populations as regard the success and failure of NIV; NIV success occurred in $\mathbf{7 1}$ out of $\mathbf{1 0 3}$ patients and the success rate was $(\mathbf{6 8 . 9 3} \%)$, NIV failure occurred in 32 patients $(\mathbf{3 1 . 0 7 \%})$ that needed intubation and invasive mechanical ventilation, the patients with failed NIV were divided according to the timing of the NIV failure into immediate failure that occurred in less than $\mathbf{1 h r}$ in $\mathbf{4}$ patients $(\mathbf{3 . 8 8 \%})$, early failure occurred during the first $\mathbf{2 4}$ hrs in $\mathbf{1 0}$ patients(9.71\%) and Late failure occurred after more than 48 hrs in $\mathbf{1 8}$ patients $(\mathbf{1 7 . 4 8 \%})$ that represent the higher number of patients with NIV failure .

Table (6): Comparison between NIV success and NIV failure as regards Duration of mechanical ventilation, Length of stay in ICU \& Hospital , Complications and Death.

\begin{tabular}{|c|c|c|c|}
\hline Variables & \begin{tabular}{|l|} 
Success \\
$\mathbf{N}=71$
\end{tabular} & \begin{tabular}{|l|} 
Failure \\
$\mathbf{N}=32$
\end{tabular} & \begin{tabular}{|l|} 
P-value \\
\end{tabular} \\
\hline $\begin{array}{l}\text { Duration of mechanic } \\
\text { ventilation } \\
\text { Mean } \\
\text { Median } \\
\end{array}$ & \begin{tabular}{|c|}
$5.40 \pm 6.33$ \\
$3(1-45)$ \\
\end{tabular} & \begin{tabular}{|l|}
$13.40 \pm 10.11$ \\
$11(1-50)$
\end{tabular} & $\mid *<0.0001$ \\
\hline $\begin{array}{l}\text { Length of stay in ICU } \\
\text { Mean } \\
\text { Median }\end{array}$ & \begin{tabular}{|l}
$7.85 \pm 7.32$ \\
$5(1-45)$
\end{tabular} & \begin{tabular}{|l|}
$17.34 \pm 17.22$ \\
$11.5(1-90)$
\end{tabular} & $\mid *<0.0001$ \\
\hline $\begin{array}{l}\text { Length of stay in Hos } \\
\text { Mean } \\
\text { Median }\end{array}$ & \begin{tabular}{|l|}
$13.85 \pm 9.86$ \\
$11(3-60)$
\end{tabular} & \begin{tabular}{|l|}
$20.00 \pm 17.48$ \\
$15(2-90)$
\end{tabular} & 0.09 \\
\hline $\begin{array}{l}\text { COMPLICATIONS } \\
\text { No } \\
\text { Yes }\end{array}$ & \begin{tabular}{|l}
$46(64.79 \%)$ \\
$25(35.21 \%)$ \\
\end{tabular} & \begin{tabular}{|l}
$4(15.5 \%)$ \\
$28(87.50 \%)$
\end{tabular} & $\mid *<0.00001$ \\
\hline $\begin{array}{l}\text { Death } \\
\text { No } \\
\text { Yes }\end{array}$ & \begin{tabular}{|l|l}
$68(95.77 \%)$ \\
$3(4.23 \%)$ \\
\end{tabular} & \begin{tabular}{|l}
$8(25.00 \%)$ \\
$24(75.00 \%)$
\end{tabular} & $\mid *<0.0001$ \\
\hline
\end{tabular}

The mean duration of mechanical ventilation, and the mean length of ICU stay were significantly lower in NIV success group in comparison to the NIV failure group, the mean length of hospitalization was longer in the NIV failed patients $(\mathbf{2 0 . 0 0} \pm \mathbf{1 7 . 4 8}$ days) but statistically not significant, the complications and the mortality rate was significantlly higher in the NIV failure patients compared to the NIV success patients( p $<0.0001$ ) (Table 6). 
SOHAG MEDICAL JOURNAL

Vol. 21 No.1 Jan 2017
Non Invasive Mechanical Ventilation in Acute Respiratory

Asmaa Ramadan khalaf

Table (7): Comparison between the NIVsuccess and NIVfailure as regards gasometric parameters:

\begin{tabular}{|c|c|c|c|c|c|c|c|c|c|c|}
\hline ABG & \begin{tabular}{|l|} 
Before \\
NIV \\
\end{tabular} & After 2 hours & \begin{tabular}{|l|}
$\begin{array}{l}\text { After 24-48 } \\
\text { hours }\end{array}$ \\
\end{tabular} & \begin{tabular}{|l|} 
Post \\
weaning
\end{tabular} & P1 & P2 & P3 & P4 & P5 & P6 \\
\hline $\begin{array}{l}\text { PH } \\
\text { Failure } \\
\text { Success }\end{array}$ & \begin{tabular}{|l}
$7.33 \pm 0$ \\
.09 \\
$7.31 \pm 0$ \\
.08
\end{tabular} & $\begin{array}{l}7.32 \pm 0.10 \\
7.35 \pm 0.07\end{array}$ & 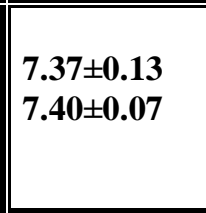 & $7.39 \pm 0.04$ & $\begin{array}{l}1.00 \\
*<0.0001\end{array}$ & $\begin{array}{l}0.42 \\
*<0.000 \\
1\end{array}$ & $*<0.0001$ & $\begin{array}{l}0.49 \\
* 0.002\end{array}$ & $* 0.004$ & $\mid 1.00$ \\
\hline$\overline{P \text { value }}$ & 0.29 & 0.13 & \begin{tabular}{|l|l}
0.14 \\
\end{tabular} & & & & & & & \\
\hline $\begin{array}{l}\text { PCO2 } \\
\text { Failure } \\
\text { Success }\end{array}$ & \begin{tabular}{|l|}
$65.09 \pm$ \\
20.84 \\
$75.11 \pm$ \\
21.40 \\
\end{tabular} & $\begin{array}{l}68.09 \pm 19.18 \\
67.03 \pm 17.36\end{array}$ & \begin{tabular}{|l}
$67.31 \pm 22.94$ \\
$61.06 \pm 15.27$
\end{tabular} & $56.76 \pm 10.18$ & $\begin{array}{l}1.00 \\
* 0.02\end{array}$ & $\begin{array}{l}1.00 \\
*<0.000 \\
1\end{array}$ & $*<0.0001$ & $\begin{array}{l}1.00 \\
0.20\end{array}$ & $* \mathbf{0 . 0 0 2}$ & $\mid 0.74$ \\
\hline P value & $* 0.03$ & 0.78 & \begin{tabular}{|l|}
0.11 \\
\end{tabular} & & & & & & & \\
\hline $\begin{array}{l}\text { PO2 } \\
\text { Failure } \\
\text { Success }\end{array}$ & \begin{tabular}{|l|}
$56.59 \pm$ \\
18.40 \\
$60.69 \pm$ \\
21.16 \\
\end{tabular} & 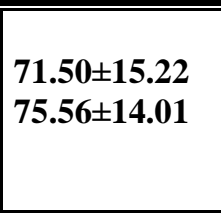 & \begin{tabular}{|l}
$73.89 \pm 22.67$ \\
$73.61 \pm 9.15$
\end{tabular} & $73.28 \pm 10.04$ & $\begin{array}{l}* 0.006 \\
*<0.0001\end{array}$ & $\begin{array}{l}* 0.002 \\
*<0.000 \\
1\end{array}$ & $*<0.0001$ & $\begin{array}{l}1.00 \\
1.00\end{array}$ & 1.00 & 1.00 \\
\hline P value & 0.35 & 0.19 & 0.93 & & & & & & & \\
\hline $\begin{array}{l}\text { SO2 } \\
\text { Failure } \\
\text { Success }\end{array}$ & \begin{tabular}{|l|}
$80.75 \pm$ \\
15.92 \\
$83.07 \pm$ \\
12.90 \\
\end{tabular} & $\begin{array}{l}91.78 \pm 3.69 \\
93.69 \pm 3.21\end{array}$ & $\begin{array}{l}90.00 \pm 11.29 \\
94.01 \pm 2.71\end{array}$ & $93.17 \pm 2.21$ & $\begin{array}{l}* 0.001 \\
1.00\end{array}$ & $\begin{array}{l}* 0.007 \\
1.00\end{array}$ & $*<0.0001$ & $\begin{array}{l}1.00 \\
1.00\end{array}$ & $*<0.0001$ & \begin{tabular}{|l|l}
$*<0$ \\
.000 \\
1
\end{tabular} \\
\hline P value & 0.44 & 0.09 & $* 0.006$ & & & & & & & \\
\hline $\begin{array}{l}\text { HCO3 } \\
\text { Failure } \\
\text { Success }\end{array}$ & \begin{tabular}{|l|}
$32.34 \pm$ \\
7.14 \\
$36.66 \pm$ \\
8.36 \\
\end{tabular} & 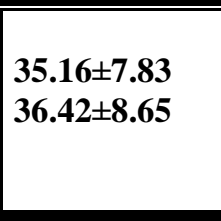 & 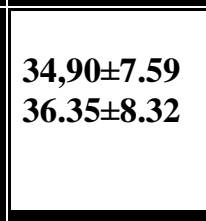 & $35.38 \pm 9.34$ & $\begin{array}{l}0.42 \\
1.00\end{array}$ & $\begin{array}{l}0.57 \\
100\end{array}$ & 1.00 & $\begin{array}{l}1.00 \\
1.00\end{array}$ & 1.00 & 1.00 \\
\hline$P$ Palue & $* 0.01$ & 0.48 & 0.42 & & & & & & & \\
\hline
\end{tabular}

- P value compare success \& failure

- p1 compared before NIV \& after $2 \mathrm{hrs}$

- p2 compared before NIV \& after 24-48hrs

- $\quad$ 3 compared before NIV \& post weaning

- $\quad$ 4 compared after $2 \mathrm{hrs} \& \&$ after $24-48 \mathrm{hrs}$

- p5 compared after $2 \mathrm{hrs} \&$ post weaning

- $\quad$ p6 compared after $24-48 \mathrm{hrs} \& \&$ post weaning

In Table (7), There is no significant differences between the NIV success and NIV failure groups as regard the baseline gasometric parameters(PH,PaO2andSaO2) ,while there is a significant increase in the baseline PaCO2, HCO3 in the success group versus the failure group.

A highly significant improvement in the parameters $\mathbf{P H}, \mathbf{P a O 2 , P a C O 2}$ in the success group $2 \mathrm{hrs}$ after NIV and during 24-48hrs of NIV, and there is a highly significant improvement in the $\mathrm{SaO} 2$ postweaning. 
In the NIV failure patients there is no significant change in the PH, PaCO2 2hrs, 2448hrs after NIV, there is initial significant improvement in $\mathbf{P a O 2}$ and $\mathrm{SaO2}$, and incomparing the gasometric parameters after $2 \mathrm{hrs}$ to $\mathbf{2 4 - 4 8 h r s ~ o f ~ N I V ~ t h e r e ~ i s ~ n o ~}$ significant change in all parameters including $\mathbf{P H}, \mathbf{P a C O 2 , P a O 2 , S a O 2 , H C O 3 .}$

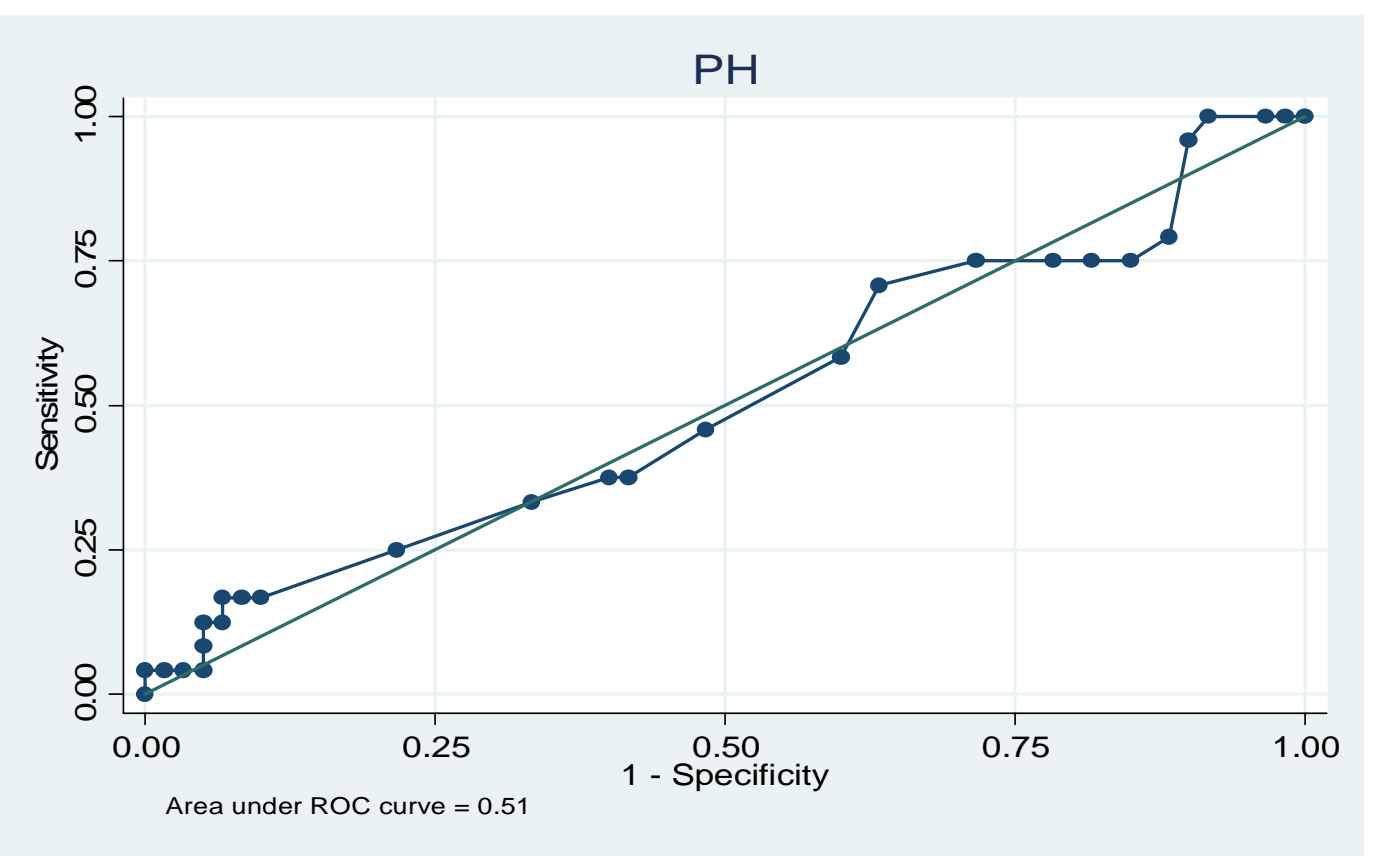

Fig (2): Area under receiving operating characteristic curve for PH to predict failure The optimum cut off level was $\leq 7.22$ with $25.0 \%$ sensitivity and $85.0 \%$ specificity, with an AUC of 0.51 (CI [0.38-0.60]).

In the present study the optimum cut off level of PH was (7.22) that predict the NIV failure outcome with more opportunity for failure below this level, it was determined using the Receiving Operating Characteristic (ROC) curve (Fig 2).

Table (8): Comparison between NIV Success and NIV Failure as regards the blood count and the electrolytes and the serum albumin level.

\begin{tabular}{|l|l|l|l||}
\hline Variables & $\begin{array}{l}\text { Success } \\
\text { N=71 }\end{array}$ & $\begin{array}{l}\text { Failure } \\
\text { N=32 }\end{array}$ & P-value \\
\hline \hline WBCs & $11.09 \pm 5.22$ & $12.20 \pm 7.32$ & 0.68 \\
\hline \hline HB & $13.11 \pm 1.97$ & $12.30 \pm 2.54$ & 0.09 \\
\hline HT & $44.14 \pm 7.52$ & $40.50 \pm 7.46$ & $* 0.02$ \\
\hline \hline PLTs & $248.86 \pm 102.16$ & $260.38 \pm 113.83$ & 0.84 \\
\hline \hline NA & $139.31 \pm 5.66$ & $137.91 \pm 6.06$ & 0.26 \\
\hline \hline K & $4.75 \pm 0.64$ & $4.84 \pm 0.80$ & 0.53 \\
\hline Mg & $2.34 \pm 0.44$ & $2.54 \pm 1.75$ & 0.52 \\
\hline \hline Ca & $9.07 \pm 0.74$ & $8.73 \pm 0.71$ & 0.07 \\
\hline \hline Albumin & $3.09 \pm 0.52$ & $2.77 \pm 0.67$ & $* 0.01$ \\
\hline
\end{tabular}

WBCs; white blood cells, HB; heamoglobin \%, HT: hematocrite value,PLTS:platelets,NA:sodium,K:potassium,Mg:magnisium,Ca:calcium 
As regard the laboratory investigations, albumin level and HT were significantly lower in the NIV failure patients and the level of the calcium, sodium and HB\% were also lower in the failure patients but statistically not significant.(Table 8).

Table (9): The clinical parameters changes before and after NIV in the study patients as regard the type of acute respiratory failure(Hypoxemic and

Hypercapnic).

\begin{tabular}{|c|c|c|c|c|c|c|}
\hline $\begin{array}{l}\text { Clinical } \\
\text { parameters }\end{array}$ & Before NIV & After 2 hours & \begin{tabular}{|l|}
$\begin{array}{l}\text { After 24-48 } \\
\text { hours }\end{array}$ \\
\end{tabular} & P1 & $\mathbf{P 2}$ & $\mathbf{P 3}$ \\
\hline $\begin{array}{l}\text { Respiratory ra } \\
\text { Hypoxemic } \\
\text { Hypercapnic }\end{array}$ & $\begin{array}{l}43.60 \pm 9.07 \\
29.49 \pm 6.03\end{array}$ & \begin{tabular}{|l|}
$30.7 \pm 7.60$ \\
$24.04 \pm 4.82$
\end{tabular} & \begin{tabular}{|l|}
$27.50 \pm 7.14$ \\
$22.67 \pm 21.75$ \\
\end{tabular} & $\begin{array}{l}* 0.004 \\
*<0.0001\end{array}$ & $\begin{array}{l}*<0.0001 \\
*<0.0001\end{array}$ & $\begin{array}{l}1.00 \\
0.22\end{array}$ \\
\hline $\begin{array}{l}\text { Heart rate } \\
\text { Hypoxemic } \\
\text { Hypercapnic }\end{array}$ & 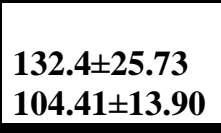 & \begin{tabular}{|l|}
$112.2 \pm 13.38$ \\
$98.68 \pm 14.39$
\end{tabular} & \begin{tabular}{|l|}
$107.00 \pm 13.90$ \\
$98.37 \pm 14.28$
\end{tabular} & $\begin{array}{l}\mathbf{0 . 0 7} \\
* \mathbf{0 . 0 2}\end{array}$ & $\begin{array}{l}* 0.02 \\
* \mathbf{0 . 0 1}\end{array}$ & $\begin{array}{l}1.00 \\
1.00 \\
\end{array}$ \\
\hline $\begin{array}{l}\text { Systolic blood } \\
\text { pressure } \\
\text { Hypoxemic } \\
\text { Hypercapnic }\end{array}$ & 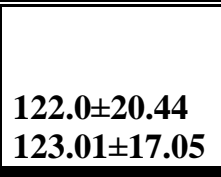 & 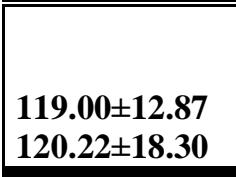 & 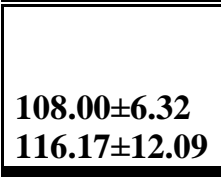 & $\begin{array}{l}1.00 \\
0.71 \\
\end{array}$ & $\begin{array}{l}\mathbf{0 . 1 2} \\
* 0.01\end{array}$ & $\begin{array}{l}0.30 \\
0.27 \\
\end{array}$ \\
\hline $\begin{array}{l}\text { Diastolic blood } \\
\text { pressure } \\
\text { Hypoxemic } \\
\text { Hypercapnic }\end{array}$ & \begin{tabular}{|l|}
$77.00 \pm 12.52$ \\
$76.24 \pm 9.99$
\end{tabular} & 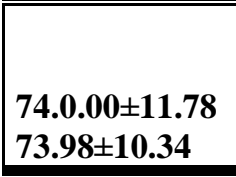 & $\begin{array}{l}68.00 \pm 4.21 \\
73.65 \pm 8.07\end{array}$ & $\begin{array}{l}1.00 \\
0.32 \\
\end{array}$ & $\begin{array}{l}0.18 \\
0.21\end{array}$ & $\begin{array}{l}0.60 \\
1.00\end{array}$ \\
\hline $\begin{array}{l}\text { Temperature } \\
\text { Hypoxemic } \\
\text { Hypercapnic }\end{array}$ & 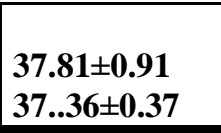 & 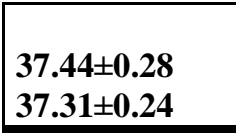 & 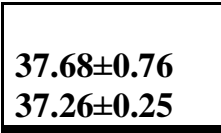 & $\begin{array}{l}0.75 \\
0.05\end{array}$ & $\begin{array}{l}1.00 \\
0.11\end{array}$ & $\begin{array}{l}1.00 \\
0.93\end{array}$ \\
\hline
\end{tabular}

- $\mathbf{p} 1$ compared before NIV \& after $2 \mathrm{hrs}$.

- 22 compared before NIV \& after 24-48hrs.

- $\quad$ 3 compared after $2 \mathrm{hrs} \&$ after 24-48hrs.

In Table (9), show a significant improvement in the clinical parameters( the respiratory rate and the heart rate) in the both types of acute respiratory failure

(the hypoxemic and the hypercapnic) after NIV (2hrs and 24-48hrs). 
SOHAG MEDICAL JOURNAL

Vol. 21 No.1 Jan 2017
Non Invasive Mechanical Ventilation in Acute Respiratory Asmaa Ramadan khalaf

Table (10): The Gasometric parameters changes before and after NIV in the study patients as regard the type of acute respiratory failure.

\begin{tabular}{|c|c|c|c|c|c|c|c|c|c|c|}
\hline $\begin{array}{l}\text { gasometric } \\
\text { parameter } \\
\text { s: }\end{array}$ & $\begin{array}{l}\text { Before } \\
\text { NIV }\end{array}$ & $\begin{array}{c}\text { After } 2 \\
\text { hours }\end{array}$ & $\begin{array}{l}\text { After 24- } \\
48 \text { hours }\end{array}$ & $\begin{array}{c}\text { Post } \\
\text { weaning }\end{array}$ & P1 & P2 & P3 & P4 & P5 & P6 \\
\hline $\begin{array}{l}\text { PH } \\
\text { Hypoxemic } \\
\text { Hypercapn } \\
\text { ic }\end{array}$ & $\begin{array}{l}7.44 \pm 0.5 \\
7.3 \pm 0.76\end{array}$ & $\begin{array}{l}7.44 \pm 0.06 \\
7.34 \pm 0.08\end{array}$ & $\begin{array}{l}7.43 \pm 0.06 \\
7.38 \pm 0.09\end{array}$ & $\begin{array}{l}7.40 \pm 0.04 \\
7.39 \pm 0.05\end{array}$ & $\begin{array}{c}1.00 \\
* 0.00 \\
6\end{array}$ & $\begin{array}{c}1.00 \\
*<0.0001\end{array}$ & $\begin{array}{c}1.00 \\
*<\mathbf{0 . 0 0 0 1}\end{array}$ & $\begin{array}{c}1.00 \\
*<0.0 \\
001 \\
\end{array}$ & $\begin{array}{c}1.00 \\
*<0.0 \\
001 \\
\end{array}$ & $\begin{array}{l}1.00 \\
1.00\end{array}$ \\
\hline $\begin{array}{l}\mathrm{PCO} 2 \\
\text { Hypoxemic } \\
\text { Hypercapn } \\
\text { ic }\end{array}$ & $\begin{array}{c}35.2 \pm 7.7 \\
1 \\
75.96 \pm 18 \\
.70\end{array}$ & $\begin{array}{c}37.10 \pm 11.5 \\
5 \\
70.61 \pm 15.4 \\
5\end{array}$ & $\begin{array}{c}39.10 \pm 10.9 \\
9 \\
65.51 \pm 16.5 \\
8 \\
\end{array}$ & $\begin{array}{l}36.17 \pm 3.18 \\
58.66 \pm 8.32\end{array}$ & $\begin{array}{l}1.00 \\
0.13\end{array}$ & $\begin{array}{c}1.00 \\
*<0.0001\end{array}$ & $\begin{array}{c}1.00 \\
*<0.0001\end{array}$ & $\begin{array}{l}1.00 \\
<0.17\end{array}$ & $\begin{array}{c}1.00 \\
*<0.0 \\
001\end{array}$ & $\begin{array}{l}1.00 \\
* 0.0 \\
46\end{array}$ \\
\hline $\begin{array}{l}\text { PO2 } \\
\text { Hypoxemic } \\
\text { Hypercapn } \\
\text { ic }\end{array}$ & $\begin{array}{c}46.30 \pm 12 \\
.81 \\
60.83 \pm 20 \\
.54\end{array}$ & $\begin{array}{c}78.30 \pm 20.1 \\
5 \\
73.87 \pm 13.7 \\
7\end{array}$ & $\begin{array}{c}79.3 \pm 13.34 \\
73.07 \pm 14.3 \\
4\end{array}$ & $\begin{array}{l}84.17 \pm 8.61 \\
72.27 \pm 9.61\end{array}$ & $\begin{array}{c}*<0.0 \\
001 \\
*<0.0 \\
001\end{array}$ & $\begin{array}{l}*<0.0001 \\
*<0.0001\end{array}$ & $\begin{array}{l}*<0.0001 \\
*<0.0001\end{array}$ & $\begin{array}{l}1.00 \\
1.00\end{array}$ & $\begin{array}{l}1.00 \\
1.00\end{array}$ & $\begin{array}{l}1.00 \\
1.00\end{array}$ \\
\hline $\begin{array}{l}\text { SO2 } \\
\text { Hypoxemic } \\
\text { Hypercapn } \\
\text { ic }\end{array}$ & $\begin{array}{c}79.60 \pm 15 \\
.08 \\
82.65 \pm 13 \\
.80\end{array}$ & $\begin{array}{l}94.00 \pm 3.97 \\
93.00 \pm 3.41\end{array}$ & $\begin{array}{c}95.80 \pm 2.39 \\
92.5 \pm 6.92\end{array}$ & $\begin{array}{l}95.33 \pm 0.52 \\
92.96 \pm 2.20\end{array}$ & $\begin{array}{l}0.93 \\
1.00\end{array}$ & $\begin{array}{l}0.66 \\
1.00\end{array}$ & $\begin{array}{l}*<0.0001 \\
*<0.0001\end{array}$ & $\begin{array}{l}1.00 \\
1.00\end{array}$ & $\begin{array}{c}*<0.0 \\
001 \\
*<0.0 \\
001\end{array}$ & $\begin{array}{l}*<0 . \\
0001 \\
*<0 . \\
0001\end{array}$ \\
\hline $\begin{array}{l}\text { HCO3 } \\
\text { Hypoxemic } \\
\text { Hypercapn } \\
\text { ic }\end{array}$ & $\begin{array}{c}23.50 \pm 4 \\
52 \\
36.59 \pm 7 \\
49\end{array}$ & $\begin{array}{c}24.3 \pm 5.93 \\
37.29 \pm 7.62\end{array}$ & $\begin{array}{c}26.30 \pm 6.18 \\
37.000 \pm 7.5 \\
9\end{array}$ & $\begin{array}{l}25.00 \pm 3.34 \\
36.34 \pm 9.14\end{array}$ & $\begin{array}{l}1.00 \\
1.00\end{array}$ & $\begin{array}{l}1.00 \\
1.00\end{array}$ & $\begin{array}{l}1.00 \\
1.00\end{array}$ & $\begin{array}{l}1.00 \\
1.00\end{array}$ & $\begin{array}{l}1.00 \\
1.00\end{array}$ & $\begin{array}{l}1.00 \\
1.00\end{array}$ \\
\hline
\end{tabular}

p1 compared before NIV \& after $2 \mathrm{hrs}$, p2 compared before NIV \& after 24-48hrs, p3 compared before NIV \& post weaning, p4 compared after 2 hrs \&after $24-48 \mathrm{hrs}$, p5 compared after $2 \mathrm{hrs} \& \&$ post weaning, , p6 compared after $24-48 \mathrm{hrs} \& \&$ post weaning

Table (10); demonstrate the changes in the gasometric parameters

(PH,PaCO2,PaO2,SaO2\%) before and after NIV in the acute Hypoxemic and hypercapnic patients. In patients with acute hypoxemic RF there is a highly significant improvement of the $\mathbf{P a O} 2$ and the $\mathbf{S a O 2 \%}$ ( $\mathbf{p}<\mathbf{0 . 0 0 0 1})$. In patients with acute hypercapnic RF there is a highly significant improvement of $\mathbf{P H}, \mathbf{P a C O 2 , P a O 2}$ and $\mathbf{S a O 2 \%}(\mathbf{p}<0.0001)$. 


\section{Discussion}

The use of noninvasive ventilation (NIV) in critically ill patients has dramatically increased(7). The recent increase in the use of NIV in the critical care units has been motivated by the desire to minimize complications of invasive ventilation. The use of NIV in treating of acute respiratory failure with diverse etiology is now supported by randomized controlled trials and metaanalysis $(\mathbf{5 , 8 , 1 0 , 1 1 , 1 3 )}$ In these studies, NIPPV was associated with a reduced need for invasive $\mathbf{M V},(\mathbf{5 , 8 , 1 0})$ decreased mortality(10-12)and shorter length of hospital stay.(8)

This study included $\mathbf{1 0 3}$ patients with a mean age of $\mathbf{5 9 . 1} \pm \mathbf{1 3 . 5}$ years, 62 males $(\mathbf{6 0 \%})$ and $\mathbf{4 1}$ females $\mathbf{( 4 0 \% )}$ admitted to respiratory intensive care unit of chest department of Assuit university hospital presented with acute respiratory failure that were managed by non invasive positive pressure ventilation, as regard the causes of ARF the study include different causes including acute exacerbations of COPD which represent $\mathbf{5 5 . 3 3 \%}$ of causes, and this is agree with randomized controlled trials and meta-analyses that support the use of NIPPV in the management of acute exacerbations of COPD, as well as ARF of other etiology(8). According to the type of respiratory failure the present study included $93(\mathbf{9 0 . 2 9 \%})$ patients presented with hypercapnic RF and 10 (9.71\%) patients presented with hypoxemic RF. The hypercapnic patients formed a significant proportion of patients in our study. Of these, $\mathbf{5 7}$ patients were diagnosed to have COPD .

This current study reports a success rate for NIV in 69\% of study patients (71 out of 103 patients). These results are consonant with previously published studies reporting success rates $\mathbf{5 0 - 8 0 \%}$ with NIV for acute respiratory failure (5,8-11).

High success has been described with NIPPV in previous studies of patients presenting with hypercapnic respiratory failure . Brochard et al. (5), observed a success rate of $\mathbf{7 4 \%}$ with NIPPV in hypercapnic patients . Martin and colleagues reported also a success rate of $\mathbf{7 8 \%}$ in the subgroup of hypercapnic patients $(\mathbf{n}=\mathbf{3 2})(\mathbf{1 3})$.

In this study, the clinical improvement of patients on NIV was confirmed by improvements in the physiological variables including $\mathbf{R R}, \mathbf{H R}, \mathbf{B P}$ within first two hours of application. There was also a significant improvement in the average $\mathbf{P H}, \mathbf{P a C O 2}$, and $\mathbf{P a O 2}$ levels within hours of application of NIV. These improvements are similar to those published in a literature in a similar population of patients(10-15).

The current study showed that $\mathbf{3 1 \%}$ of the patients demonstrated NIV failure which agree with other rates reported in other studies $(\mathbf{9 , 1 6 )}$ that showed NIV failure rates between $\mathbf{7 \%}$ and $\mathbf{6 2 . 5 \%}$. The failure group had significantly longer duration of mechanical ventilation, ICU and hospital length of stay and had significantly higher complications and mortality rate. In this study the success group included only $\mathbf{2 5}$ patients had complications while 46 had no complications in which that results was highly significant in comparison to the failure group in which $\mathbf{2 8}$ out of $\mathbf{3 2}$ patients had complications $(\mathbf{p}$ value $=$ 0.00001) and this in agreement with Girou et al (22) and Gay (23) in which NIV reduces by three to five times the risk of pneumonia associated with IMV, especially in immunosuppressed patients and those with comorbidities. 
Our study found a PH of $\mathbf{7 . 2 2}$ was a cutoff point in determining success or failure with more opportunity for failure below this level, this was similar to some centres describe that the most beneficial effect is obtained at level of PH less than 7.30(14), while some centres report success at a worse $\mathrm{PH}$ value like $\mathbf{7 . 2 5}$ and less(18), even 7.13(19).

The present study results as regard laboratory investigations in comparison between success and failure groups show a significant lower level of serum albumin in the failed $\operatorname{group}(\mathbf{p}=\mathbf{0 . 0 1})$. Vitacca et al (20)and Boosalis et al.,(21) reported similar conclusions in a study of 39 COPD patients. They found that malnourished patients, estimated by anthropometry, had higher risk of having poor outcome and increased need of MV In the current study, the survivors percentage of studied population was $\mathbf{7 4 \%}$, the mortality rate of studied population was $26 \%$ included $23 \%$ ICU mortality and $\mathbf{3 \%}$ hospital mortality .The mortality rate was highly significantly

\section{Referances}

1. Palange P., Simonds A.K.: ERS Handbook of Respiratory MedicineDefinition of Respiratory Failure, 2010 .

2. Cartin-Ceba R, Kojicic M, Li G.: Epidemiology of critical care syndromes, organ failures, and lifesupport interventions in a suburban US community. Chest. 2011;140(6):144755.

3. Demoule A, Chevret S, Carlucci A.: Changing use of noninvasive ventilation in critically ill patients: trends over $\mathbf{1 5}$ years in francophone countries. Intensive Care Med 2016 ;42:82-92.

4. Nava S, Hill $\mathrm{N} \quad \therefore$ Non-invasive ventilation in acute respiratory higher in the NIV failed group $\mathbf{( 7 5 . 0 0 \% )}$ in comparison to the NIV success group $(4.23 \%)$ ( $p$ value $<0.0001)$. this was similar to Singh et al.,(2006) reported that in patients with acute respiratory failure, associated with NIV failure show increased mortality(24).

\section{Conclusion}

The use of noninvasive ventilation (NIV) in patients with acute respiratory failure has shown to be effective in the relief of dyspnea, the improvement of vital signs and gas exchange, in preventing endotracheal intubation, and improving overall survival.

Monitoring of patients closely is necessary to evaluate treatment responsiveness and to facilitate endotracheal intubation if NIV fails.

As a predicted parameter of NIV failure; $\mathrm{PH}$ of $\mathbf{7 . 2 2}$ was a cut-off point .

\section{Conflict of interest}

We have no conflict of interst to declare

failure,Lancet $\quad 2009 ; \quad 374(9685): 250$ 259.

5. Brochard L, Mancebo J, Wysocki M, Lofaso F, Conti G, Rauss A, Noninvasive ventilation for acute exacerbations of chronic obstructive pulmonary disease. $\mathrm{N}$ Engl J Med 1995;333(13):817-822.

6. Gray A, Goodacre S, Newby DE, Masson M, Sampson F, Nicholl J. Noninvasive ventilation in acute cardiogenic pulmonary edema. N Engl J Med. Jul 10 2008; 359(2):142-51

7. Demoule A, Chevret S, Carlucci A et al (2016) Changing use of noninvasive ventilation in critically ill patients: trends over 15 years in 
francophonecountries. Intensive Care Med 42:82-92

8. Ram FS, Lightowler JV, Wedzicha JA.: Non-invasive positive pressure ventilation for treatment of respiratory failure due to exacerbations of chronic obstructive pulmonary disease. Cochrane Database Syst Rev 2004;(1):CD004104.

9. Mohamed A. Zamzam, Amal A. Abd El Aziz, Maha Y. Elhefnawy, Nagia A. Shaheen :Study of the characteristics and outcomes of patients on mechanical ventilation in the intensive care unit of EL-Mahalla Chest Hospital, Egyptian Journal of Chest Diseases and Tuberculosis 2015; 64, 693-701.

10. 4. Peter JV, Moran JL, Phillips-Hughes J, Graham P, Bersten AD. Effect of non-invasive positive pressure ventilation (NIPPV) on mortality in patients with acute cardiogenic pulmonary oedema: A metaanalysis. Lancet 2006;367:1155-63.

11. Peter JV, Moran JL, Phillips-Hughes J, Warn D.: Non-invasive ventilation in acute respiratory failure: a metaanalysis update.Crit Care Med 2002;30:555-62.

12. Plant PK, Owen JL, Elliott MW. Early use of noninvasive ventilation for acute exacerbations of chronic obstructive pulmonary disease on general respiratory wards: A multicentre randomized controlled trial. Lancet 2000;355:1931-5.

13. Martin TJ, Hovis JD, Costantino JP, Bierman MI, Donahoe ME, Rogers $\mathrm{RM}$, et al. A randomized, prospective evaluation of noninvasive ventilation for acute respiratory failure. Am $\mathrm{J}$ Respir Crit Care Med 2000;161:80713.

14. Keenan SP, Sinuff T, Cook DJ, Hill NS. Which patients with acute exacerbation of chronic obstructive pulmonary disease benefit from noninvasive positive-pressure ventilation? A systematic review of the literature, Ann Intern Med. 2003 ;138(11):861-70.

15. Khilnani GC, Saikia N, Sharma SK, Pande JN, Malhotra OP. EfP cacy of noninvasive pressure ventilation for the management of COPD with acute or acute on chronic respiratory failure: A randomized controlled trial. Am J Respir Crit Care 2002;165:A387

16. Lightowler J.V., Wedjicha J.A., Elliott M.W.: Noninvasive positive pressure ventilation to treat respiratory failure resulting from exacerbations of chronic obstructive pulmonary disease: cochrane systematic review and meta analysis, BMJ 326 2003; 185-187.

17. Keenan SP, Sinuff T, Cook DJ, Hill NS.: Does noninvasive positive pressure ventilation improve outcome in acute hypoxemic respiratory failure? A systematic review. Crit Care Med. 2004; 32:2516-23.

18. Confalonieri M, Garuti G, Cattaruzza MS, Osborn JF, Antonelli M, Conti G,Kodric M, Resta O, Marchese S, Gregoretti C, Rossi A.: A chart of failure risk for noninvasive ventilation in patients with COPD exacerbation, Eur Respir J 2005; 25:348-355.

19. Diaz GG, Alcaraz AC, Talavera JC, Noninvasive positive-pressure ventilation to treat hypercapnic coma secondary to respiratory failure, Chest. 2005; Mar. 127(3):952-60.

20. Vitacca M, Clini E, Porta R, Foglio K, Ambrosino N.: Acute exacerbations in patients with COPD: Predictors of need for mechanical ventilation. Eur Respir J 1996;9:1487-93. 
21. Boosalis MG, Ott L, Levine AS, Slag MF, Morley JE, Young B,: Relationship of visceral proteins to nutritional status in chronic and acute stress. Crit Care Med 1989;17:741-7.

22. Girou E, Schortgen F, Delclaux C.: Association of noninvasive ventilation with nosocomial infections and survival in critically ill patients. J Am Med Assoc 2000; 284: 2361-2370.
23. Gay PC. Complications of noninvasive ventilation in acute care. Respir Care 2009; 54: 246-57.

24. Singh VK, Khanna P, Rao BK, Sharma SC, Gupta R.: Outcome predictors for noninvasive positive pressure ventilation in acute respiratory failure. $\mathrm{J}$ Assoc Physicians India 2006; 54: 361-5. 
SOHAG MEDICAL JOURNAL

Vol. 21 No.1 Jan 2017 